pag

Business School

WORKING PAPER SERIES

Working Paper

$$
\text { 2014-156 }
$$

Carbon price analysis using empirical mode decomposition

Bangzhu Zhu

Ping Wang

Julien Chevallier

Yiming Wei

http://www.ipag.fr/fr/accueil/la-recherche/publications-WP.html

IPAG Business School

184, Boulevard Saint-Germain

75006 Paris

France 


\title{
Carbon price analysis using empirical mode decomposition
}

\author{
Bangzhu Zhu ${ }^{\mathrm{a}, \mathrm{b}}$, Ping Wang ${ }^{\mathrm{a}}$, Julien Chevallier ${ }^{\mathrm{c}^{*}}$, Yiming Wei ${ }^{\mathrm{b}}$ \\ ${ }^{a}$ School of Economics and Management, Wuyi University, Jiangmen, Guangdong 529020, China \\ ${ }^{b}$ Center for Energy and Environmental Policy Research, Beijing Institute of Technology, Beijing 100081, China \\ ${ }^{c}$ IPAG Business School, IPAG Lab, 184 Boulevard Saint-Germain, 75006 Paris, France
}

\begin{abstract}
Mastering the underlying characteristics of carbon price changes can help governments formulate correct policies to keep efficient operation of carbon markets, and investors take effective measures to evade their investment risks. Empirical mode decomposition (EMD), a self-adaption data analysis approach for nonlinear and non-stationary time series, can accurately explain the formation mechanism of carbon price by decomposing it into several intrinsic mode functions (IMFs) and one residue from different scales. In this study, we apply EMD to the European Union Emissions Trading Scheme (EU ETS) carbon price analysis. First, the carbon price is decomposed into eight IMFs and one residue. Moreover, these IMFs and residue are reconstructed into a high frequency component, a low frequency component and a trend component using hierarchical clustering method. The economic meanings of these three components are identified as short term market fluctuations, effects of significant trend breaks, and a long-term trend, respectively. Finally, some strategies are proposed for carbon price forecasting.
\end{abstract}

Keywords: Carbon Price; Empirical Mode Decomposition; Multiscale Analysis; Forecasting; EU ETS

\section{Introduction}

On February 16, 2005, the Kyoto Protocol came into force, which states that the European Union (EU) is committed to reducing greenhouse gas emissions by $8 \%$ below its 1990 level during the period 2008-2012. In order to meet the commitment at the lowest overall costs, also in 2005, the EU Emissions Trading Scheme (EU ETS) was launched within the EU covering around 12,000 installations in 25 countries and 6 major industrial sectors. In terms of market value and trading volume, the EU ETS is the largest carbon market all over the world up to date [1]. Therefore, we focus on the EU ETS carbon price analysis in this study.

During the past few years, there have been some studies on the analysis and forecasting of EU ETS carbon prices. These empirical approaches can be grouped into two categories: structured models and data-driven models. The structured models outline the carbon markets characteristics, and then analyze the carbon price movements mainly from the perspective of supply-demand equilibrium [1, 2]. Data-driven models include linear models such as Multivariate Linear Regression (MLR), Autoregressive Moving Average (ARMA), Vector Autoregressive (VAR), Granger Causality Test, Autoregressive Conditional Heteroscedasticity (ARCH), Generalized ARCH (GARCH), Threshold GARCH (TGARCH) and Markov Switching-AutoRegressive-GARCH models [3-5], and nonlinear models such as Differential Equation [6], Rescaled Range (R/S) analysis [7]. There are a number of other references on this topic [8-19].

The structured models can help us understand the generation of carbon prices, and estimate each factor's impact on carbon prices. However, this approach has proved to be difficult to be implemented in practice, because

\footnotetext{
* Corresponding author. Address: Tel.: +33 (0)149407386; fax: +33 (0)149407255. E-mail address: julien.chevallier04@univ-paris8.fr (J. Chevallier).
} 
that it is hard to model the supply and demand under the dynamic and unstable market environment. Data-driven methods often perform well for short-term carbon price forecasting, but they lack the economic meaning and cannot well explain the inner driving forces of carbon price movements.

Empirical mode decomposition (EMD) [20], a self-adaption data analysis approach for nonlinear and non-stationary time series, proposed by Huang et al. in 1998, can solve the dilemma between the difficulties in modeling and the lack of economic meaning. EMD can decompose carbon prices into several independent intrinsic mode functions (IMFs) and one residue based on local characteristic scales [21]. Thus, we can identify the concrete implications of each IMF and the residue according to its own scale. For example, an IMF with a scale of three months can be recognized as the seasonal component. Since the carbon price is the only link we have with the reality, via exploring data's IMFs, EMD can help not only discovering the characteristics of carbon prices but also understanding the underlying rules of carbon prices. Therefore, EMD is effective for nonlinear and non-stationary carbon price analysis.

EMD was initially proposed for the study of ocean waves, and then successfully applied in many areas, including biomedical engineering, structured health monitoring and earthquake engineering. In recent years, there have been some successful applications in social sciences so far. Huang et al. applied EMD to financial data to examine the changeability of the markets [22]. Zhang et al. adopted EMD for West Texas Intermediate (WTI) crude oil spot price analysis [23]. Yu et al. applied EMD for financial crisis forecasting [24]. Zhu applied EMD for carbon price forecasting [25]. Moghtaderi et al. analyzed trend filtering by EMD with applications to environmental studies and bicycle rental services [29]. In this study, we will show that EMD can be useful for carbon price analysis.

The contributions of this paper are twofold. Firstly, we carry out an EMD-based multiscale analysis for the carbon price which is decomposed into several IMFs and one residue, from high to low frequency. These IMFs and residue are reconstructed into a high frequency component, a low frequency component and a trend component by hierarchical clustering method. The economic meanings of these three components are identified as short term fluctuations, effects of significant trend breaks, and a long-term trend, respectively. Secondly, according to the results of the carbon price analysis, we propose some forecasting strategies for carbon prices. All components are predicted based on their own features, and then integrate their results to obtain a final result of the original carbon price. Numerical details on the computational steps to obtain the results in the Matlab 2013a software are included in the paper.

The main motivation of this study is to apply EMD for carbon price analysis and attempt to interpret the formation mechanism of carbon price from a novel perspective. The rest of this paper is organized as follows: Section 2 outlines EMD and hierarchical clustering method; Section 3 introduces the EU ETS carbon price data and decomposes them by EMD. The derived IMFs are also shown in this section. Section 4 presents some analyses based on a composition using the hierarchical clustering method. Section 5 concludes this paper.

\section{Methodology}

\subsection{EMD}

EMD assumes that the carbon price simultaneously has many IMFs of different oscillations. Each IMF can be extracted from carbon price based on local characteristic scale of carbon price itself. IMF meets two conditions 
[20]: (1) IMF has the same number of extremum and zero-crossings or differs by one at the most; (2) IMF is symmetric with the local zero mean. EMD can extract the IMFs through a sifting process as follows:

(1) Identify all the maxima and minima of carbon price data $x(t)$;

(2) Generate their upper and lower envelopes, $e_{\max }(t)$ and $e_{\min }(t)$, with cubic spline interpolation;

(3) Calculate the point-by-point mean $m(t)$ from the upper and lower envelopes:

$$
m(t)=\frac{e_{\max }(t)+e_{\min }(t)}{2}
$$

(4) Extract the mean from carbon price data and define the difference between $x(t)$ and $m(t)$ as $d(t)$ :

$$
d(t)=x(t)-m(t)
$$

(5) Check the properties of $d(t)$ :

(1) If it is an IMF, denote $d(t)$ as the $i$ th IMF and replace $x(t)$ with the residue $r(t)=x(t)-d(t)$. The $i$ th IMF is often denoted as $c_{i}(t)$ and the $i$ is called its index;

(2) If it is not an IMF, replace $x(t)$ with $d(t)$;

(6) Repeat steps (1) - (5) until the residue satisfies some stopping criteria. One typical stopping criterion proposed by Rilling et al. [26] introduces the mode amplitude $\alpha(t):=\frac{e_{\max }(t)-e_{\min }(t)}{2}$, and the evaluation function $\sigma(t):=\left|\frac{m(t)}{\alpha(t)}\right|$. Thus the sifting is iterated until $\sigma(t)<\theta_{1}$ for some prescribed fractions $(1-\alpha)$ of the total duration, while $\sigma(t)<\theta_{2}$ for the remaining fractions, where $\theta_{1}$ and $\theta_{2}$ are two thresholds aimed to guarantee globally small fluctuations in the mean while taking into account locally large excursions. One can typically set $\alpha=0.05, \theta_{1}=0.05$ and $\theta_{2}=0.5$.

At the end of this sifting procedure, the carbon price $x(t)$ can be expressed as

$$
x(t)=\sum_{i=1}^{m} c_{i}(t)+r_{m}(t)
$$

where $m$ is the number of IMFs, $r_{m}(t)$ is the final residue, and $c_{i}(t)$ represents the $i$ th IMF.

\subsection{Hierarchical clustering method}

The main aim of clustering analysis is to identify variables on some features of similar or dissimilar, and according to these features variables are divided into several categories. There are many clustering analysis methods, among which hierarchical clustering method [27] is one of the most commonly used.

After decomposing the carbon price into $m+1$ IMFs (including one residue) by EMD, a high frequency component, a low frequency component and a trend component can be obtained from the hierarchical clustering method:

(1) Calculate the distance between any two IMFs, which can be selected from the Euclidean distance, Chebychev distance, Minkowski distance, and so on.

(2) Divide each IMF into a category, and get $m+1$ categories.

(3) Merge the two categories of minimum distance into a new category. 
(4) After merging, when the number of categories is equal to 1, go to (5), otherwise calculate the current distances between the new category and the other categories, and then return to (3).

(5) Draw the clustering tree, and then according to the practical requirements and the shape of clustering spectrum diagram, determine the reasonable classification results.

(6) According to the classification results, compose the corresponding IMFs into a high frequency component, a low frequency component and a trend component.

\section{Decomposition}

\subsection{Data}

The European Climate Exchange (ECX) located in London, UK, is the largest carbon exchange market within the EU ETS, since its daily carbon trading volume generally accounts for over $80 \%$ of the total carbon trading volume of EU ETS. It goes without saying that the ECX can largely reflect the overall state and trends of the EU ETS.

The daily data used is the ECX carbon future price matured in December, 2013, i.e., DEC 13, which is one of the main future contracts currently traded in the carbon market. Fig.1 illustrates DEC13 from April 8, 2008 to June 21, 2013, with a total of 1,335 Transaction day (TD) data points in unit of Euros/ton, which shows that carbon price movements appear to be nonlinear and nonstationary in that the means are changing over time.

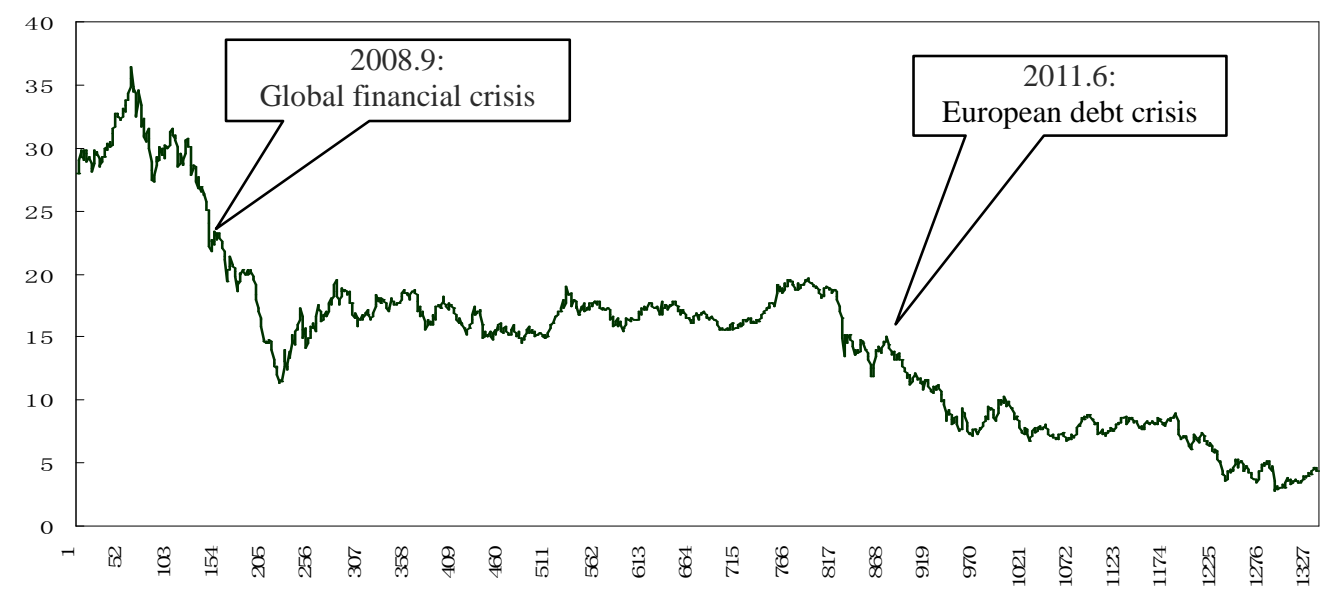

Fig. 1. DEC13 from April 8, 2008 to June 21, 2013.

\subsection{IMFs}

The EMD was implemented in the Matlab R2013a software package produced by the MathWorks Inc, and run on a personal computer with an Intel Core i3-2130 Duo CPU 3.40 GHz and 2.0 GB RAM. Among the abundant Matlab functions library, we have used the find and diff functions to find out all the local maximal and minimal positions and values of the carbon price, and the spline function to form the cubic spline interpolation. In the meantime, the stopping criterion was set as $\alpha=0.05, \theta_{1}=0.05$ and $\theta_{2}=0.5$. Fig. 2 shows that the carbon price is decomposed into eight IMFs plus one residue via EMD. All the IMFs are listed from high to low frequency, and the last one is the residue.

The frequencies and amplitudes of all the IMFs vary over time. As the frequency changes from high to low, the amplitudes of the IMFs are becoming larger: for example, most the amplitudes of IMF1 are smaller than 1, but the amplitudes of IMF8 are limited to 6 . The residue slowly varies around the long term mean. 


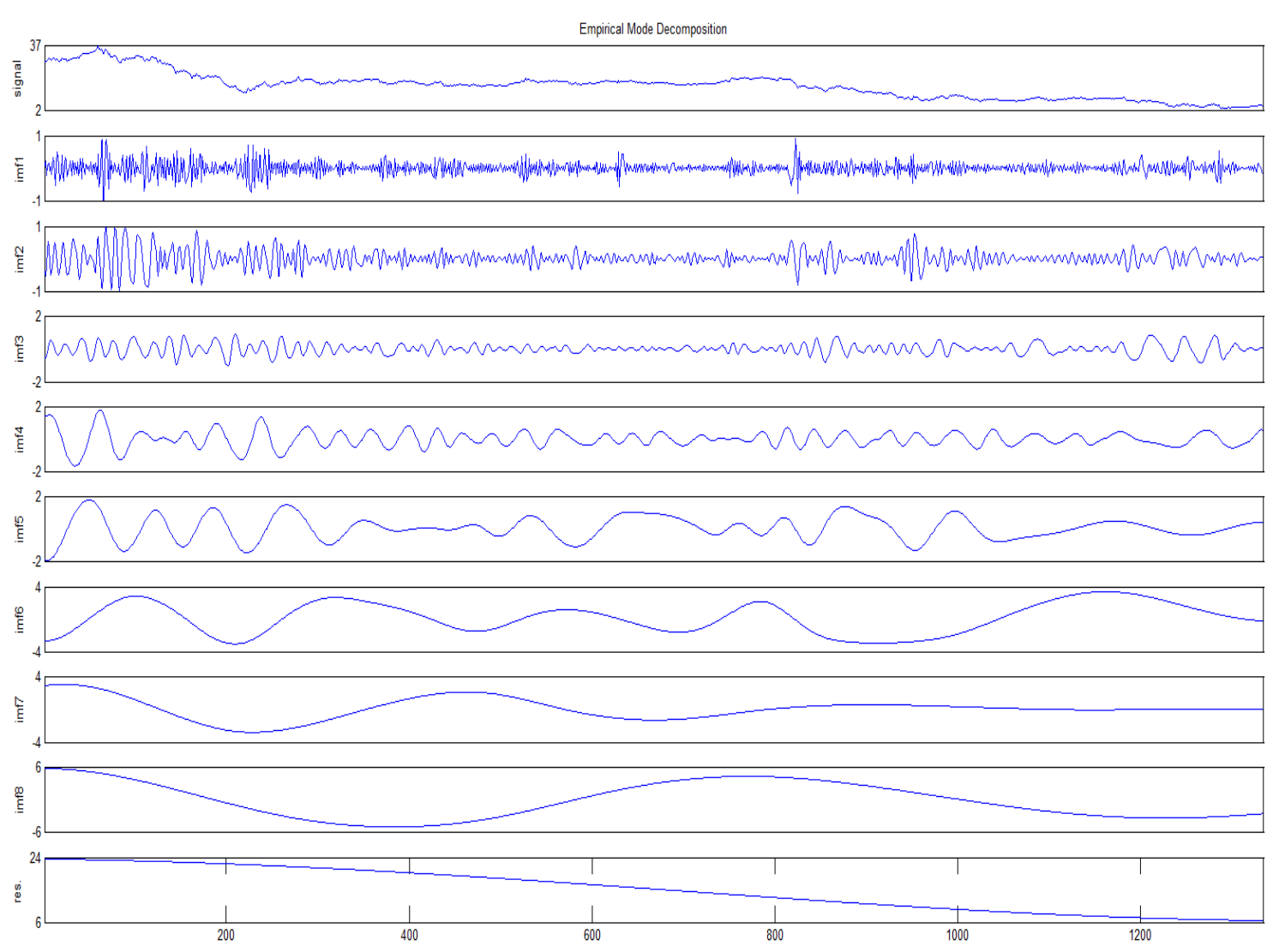

Fig. 2. IMFs and residue for DEC13 derived from EMD.

\subsection{IMF statistics}

We take some measures to analyze the IMFs: the mean period of each IMF, the correlation between each IMF (residue) and the original carbon price, the variance and variance percentage of each IMF. Table 1 shows related information about this decomposition.

Table 1. Measures of IMFs and residue for DEC13 derived from EMD

\begin{tabular}{|c|c|c|c|c|c|c|}
\hline & $\begin{array}{c}\text { Mean } \\
\operatorname{period}(T D)\end{array}$ & $\begin{array}{l}\text { Pearson } \\
\text { correlation }\end{array}$ & $\begin{array}{c}\text { Kendall } \\
\text { correlation }\end{array}$ & Variance & $\begin{array}{c}\text { Variance as } \\
\% \text { of the } \\
\text { observed }\end{array}$ & $\begin{array}{c}\text { Variance as } \\
\% \text { of ( } \Sigma \text { IMFs } \\
\text { +residue })\end{array}$ \\
\hline Observed & & & & 50.241 & & \\
\hline IMF1 & 6.51 & 0.032 & 0.02 & 0.038 & 0.076 & 0.076 \\
\hline IMF2 & 14.75 & 0.023 & 0.018 & 0.063 & 0.125 & 0.127 \\
\hline IMF3 & 32.56 & $0.067^{*}$ & $0.065^{* *}$ & 0.104 & 0.207 & 0.209 \\
\hline IMF4 & 76.29 & $0.102^{* *}$ & $0.116^{* *}$ & 0.249 & 0.496 & 0.500 \\
\hline IMF5 & 178 & $0.118^{* *}$ & $0.115^{* *}$ & 0.509 & 1.013 & 1.022 \\
\hline IMF6 & 534 & 0.02 & $0.098^{* *}$ & 3.516 & 6.998 & 7.062 \\
\hline IMF7 & 667.5 & $0.247^{* *}$ & $0.045^{*}$ & 1.653 & 3.290 & 3.320 \\
\hline IMF8 & 2670 & $0.512^{* *}$ & $0.290^{* *}$ & 10.739 & 21.375 & 21.569 \\
\hline Residue & - & $0.851^{* *}$ & $0.675^{* *}$ & 32.918 & 65.520 & 66.115 \\
\hline Sum & & & & & 99.100 & 100 \\
\hline
\end{tabular}

*: Correlation is significant at the level of 0.05 (2-tailed); **: Correlation is significant at the level of 0.01 (2-tailed).

We define the mean period as the value derived from dividing the number of points by the number of peaks for each IMF, since the frequency of an IMF may change with time continuously and the periods are also not constant. When an IMF is $T$ long and has $s$ peaks, the mean period of such an IMF is $T / s$. We employ the Pearson product moment correlation coefficient and Kendall rank correlation coefficient to simultaneously 
measure the correlations between each IMF (residue) and the observed carbon price. Since these IMFs (residue) are independent of each other, we use the percentage of variance to explain the contribution of each IMF (residue) to the total movement of the observed carbon price. However, due to some limitations [28], the variances of IMFs and residue do not always add up to the total variance, for example, there is a negative $0.9 \%(99.1 \%-100 \%)$ difference in Table 1.

As shown in Table 1, we can find out that the residue is the dominant mode of the carbon price. Both Pearson and Kendall coefficients, between the residue and the original carbon price, reach a high level of more than 0.851 and 0.675 in Table 1, respectively, both with significance at the level of 0.01 . At the same time, the variance of the residue accounts for $66.115 \%$ of the total variance. The IMF6, IMF7 and IMF8 are the other important modes with a mean period of more than one year. Meanwhile, the sum of variances for these important components, the IMF6, IMF7, IMF8 and residue, contribute $98.066 \%$ of the total variance. On the other hand, the first five IMFs not only exhibit very low correlation coefficients with the original carbon price, but also account for only $1.934 \%$ of total variance, which means that these IMFs have no serious effect on carbon price changes.

\section{Composition}

The hierarchical clustering analysis was also implemented in the Matlab R2013a software package. Among the library of Matlab functions, we have used the pdist function to compute the Euclidean distance between pairs of any two IMFs, or any an IMF and the residue; the linkage function to create a hierarchical cluster tree using the smallest distance principle; and the dendrogram function to generate a dendrogram plot of the hierarchical cluster tree. Fig. 3 gives the clustering results. After careful analysis, the eight IMFs and the residue prefer to be grouped into three categories: (i) the first one: the Euclidean distance is smaller than 6; (ii) the second one: the Euclidean distance lies between 6 and 12; and (iii) the third one: the Euclidean distance is more than 12. Therefore, in terms of the mean periods, the partial reconstruction with IMF1, IMF2, IMF3, IMF4 and IMF5 can be recognized as the high frequency component, the partial reconstruction with IMF6, IMF7 and IMF8 can be treated as the low frequency component, and the residue can be served separately as the trend component. Fig. 4 shows the three components and Table 2 gives statistical measures.

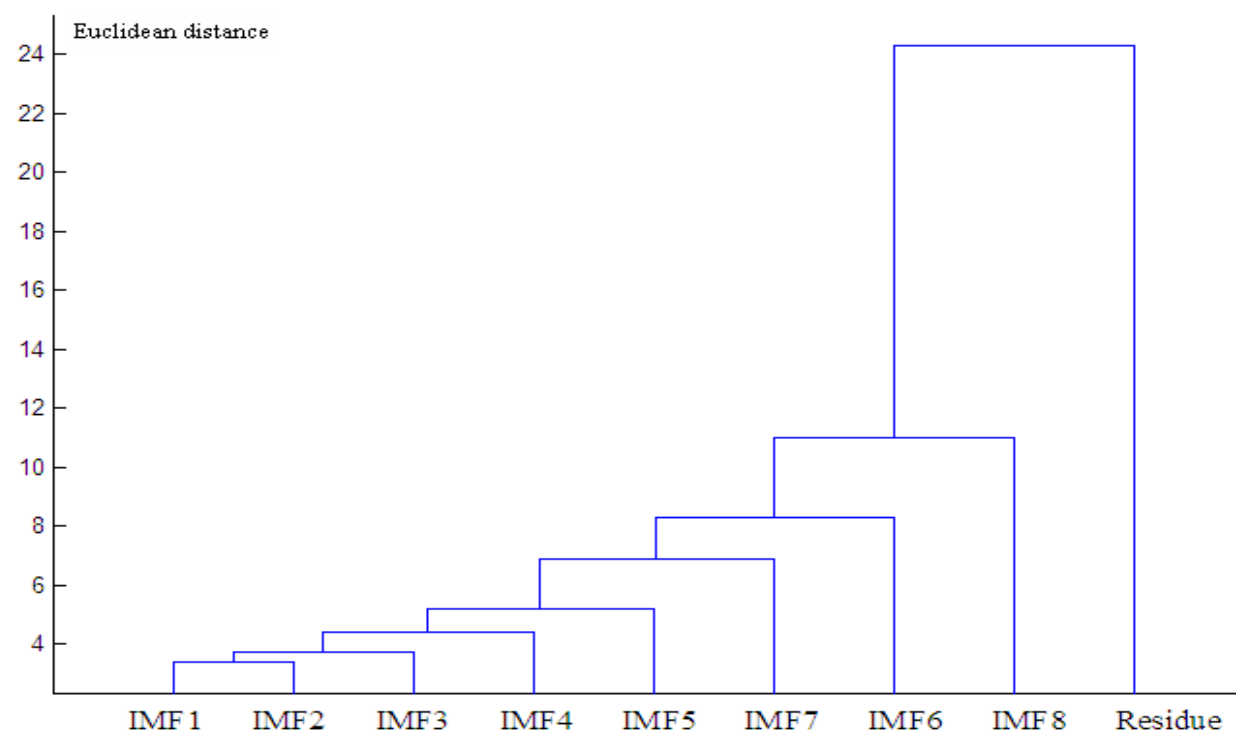

Fig. 3. Hierarchical clustering tree derived from the IMFs and residue. 
Each component has its own distinct features. The residue slowly varies around the long term average. Thus, it is considered as the long term trend during the evolution of the carbon price; every sharp up or down of the low frequency component corresponds to a significant trend break, which is an indicator of the effects of a significant trend break; and the high frequency component reflects the effects of short term market fluctuations.

For the movement trend of the observed carbon price, the most important components are low frequency component and trend. Pearson and Kendall correlation coefficients between the low frequency component, trend component and the original carbon price reach $0.572,0.851,0.292$ and 0.675 , respectively, all with significance at the level of 0.01; meanwhile, low frequency components and trends have higher variance contributions, amounting for $27.146 \%$ and $70.706 \%$, respectively. The sum of variances for these two important components, low frequency component and trend component, contribute $97.852 \%$ of total variance. Meanwhile, the high frequency component has a small effect on the whole changes of the original carbon price.

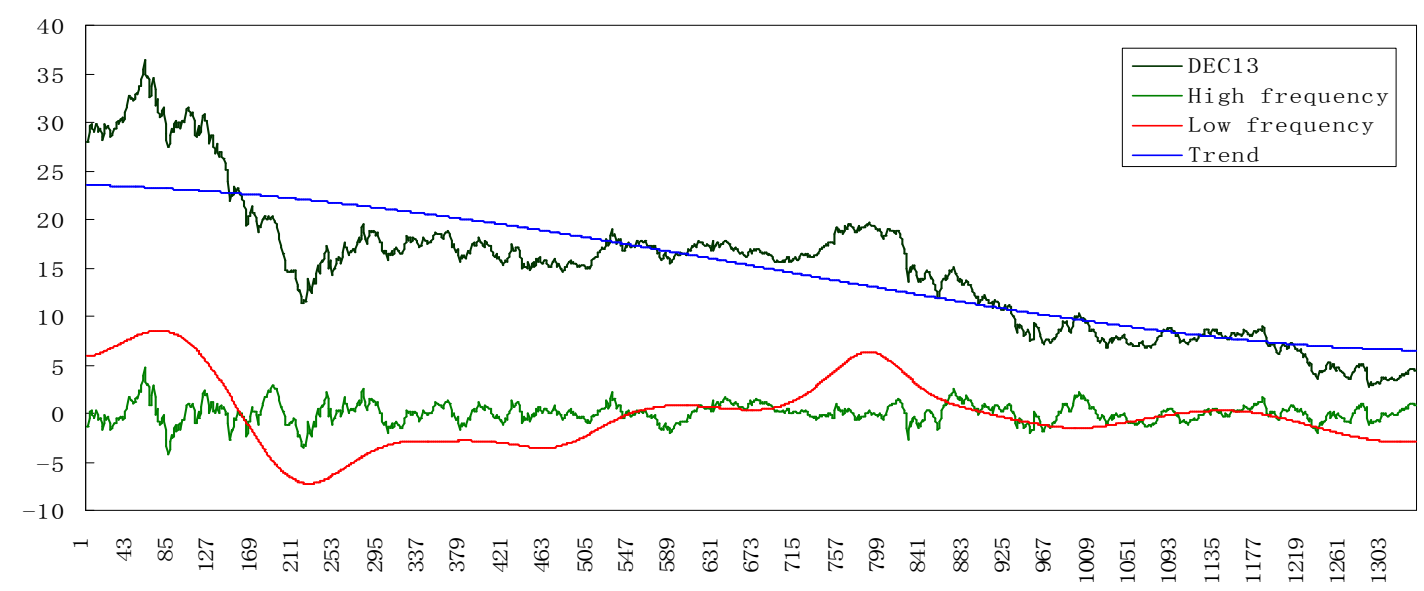

Fig. 4. Three components of DEC13.

Table 2. Correlation and variance of components for DEC11

\begin{tabular}{ccccccc}
\hline & $\begin{array}{c}\text { Mean period } \\
\text { (TD) }\end{array}$ & $\begin{array}{c}\text { Pearson } \\
\text { correlation }\end{array}$ & $\begin{array}{c}\text { Kendall } \\
\text { correlation }\end{array}$ & Variance & $\begin{array}{c}\text { Variance as } \\
\% \text { of the } \\
\text { observed }\end{array}$ & $\begin{array}{c}\text { Variance as } \% \\
\text { of }(\Sigma \text { IMFs } \\
\text { +residue })\end{array}$ \\
\hline $\begin{array}{c}\text { Observed } \\
\text { High frequency } \\
\text { component } \\
\text { Low frequency } \\
\text { component } \\
\quad \begin{array}{c}\text { Trend } \\
\text { Sum }\end{array}\end{array}$ & 8.116 & $0.169^{* *}$ & $0.160^{* *}$ & $\begin{array}{c}50.241 \\
1.000\end{array}$ & & \\
& 445 & $0.572^{* *}$ & $0.292^{* *}$ & 12.638 & 1.9904 & 2.148 \\
& - & $0.851^{* *}$ & $0.675^{* *}$ & 32.918 & 65.52 & 70.706 \\
\end{tabular}

**: Correlation is significant at the level of 0.05 (2-tailed).

\subsection{Trend}

The trend has a high correlation with the original carbon price and contributes more than $70 \%$ of the total variance of the original carbon price. Therefore, the trend is a crucial factor for the long-term movement of carbon price, and a deterministic force for the carbon price evolution in the long run. The evolution of the trend as a whole is consistent with the economic development in the EU, declaring that the long term trend of the carbon price is determined by EU economic development. The trend appears to decline, which is related to the fact that the global financial crisis since September, 2008, especially the European debt crisis since June, 2011 dragged down the carbon price.

By comparing the trend with the original carbon price, we can find that although the carbon price will fluctuate dramatically due to significant trend breaks, it will return to the trend after the influence of the trend 
breaks faded away. For example, the global financial crisis since September, 2008 made carbon price fall suddenly from over 35 Euros/ton to below 15 Euros/ton, but the price rose slowly after that and finally returned to the trend price of about 20 Euros/ton in May, 2009.

\subsection{Effects of significant trend breaks}

The significant trend breaks such as intergovernmental negotiations, national allocation plans and global financial crisis can seriously affect the carbon price. Because the occurrences of significant trend breaks are not frequently, their effects are mainly embodied in the low frequency component derived from IMF6 to IMF8. In terms of the mean periods of these IMFs, the shortest is about two years and the longest is more than ten years, demonstrating that it is very difficult for the carbon market itself to eliminate these effects soon; a significant trend break can affect the carbon price for a long time. In addition, the amplitudes at some time points could be more than 4 Euros/ton or even higher, showing that the effects of trend breaks on the carbon price can be very serious. Therefore, significant trend breaks can lead to large fluctuations in medium term.

Through separating the effects of significant trend breaks included in the low frequency component from the whole carbon price, we can the measure the effect of each significant trend break used as a reference for predicting the effect of the next significant trend break of the same or similar type. For example, during the period of the global financial crisis beginning from September, 2008, carbon price fell to 10 Euros/ton or below from the summit more than 20 Euros/ton in summer, with a decline of over 10 Euros/ton, which roughly means that the maximum effect of this trend break on the carbon price was 10 Euros/ton or above, and the effects of this trend break have continued until present.

\subsection{Normal market fluctuations}

Besides significant trend breaks and the long-term trend, the carbon price is also influenced by many other factors, such as extreme weather conditions and speculations $[1,3,4,9,12]$. Durations of these effects are often short. So they are classified into high frequency trend breaks and their effects are contained in the high frequency component. The IMF1, IMF2, IMF3, IMF4, and IMF5 have shorter mean periods and make little variance contribution, proving that the normal market fluctuations have no serious impact on the carbon price - it is in general less than 2 Euros/ton. However, these trend breaks are becoming more and more frequent and have lately become the fundamental forces of carbon price changes. Thus, normal market fluctuations can be neglected in long-term forecasting, but they are important for short-term forecasting.

In brief, the carbon price is composed of three components: the high frequency component, the low frequency component and the residue. The residue shows the major trend of carbon price in the long-term. The low frequency component expresses the effects of significant trend breaks which have a medium-term impact on carbon price. The high frequency component displays normal market fluctuations which have only a short-term impact on carbon price. Through this method, the carbon price of 30 Euros/ton in August, 2008 can be decomposed into a trend price (about 23 Euros/ton), the effect of a significant trend break (about 8 Euros/ton) and short-term market fluctuations (about -1 Euros/ton); and the carbon price of 8 Euros/ton in August, 2012 can be decomposed into a trend price (about 8 Euros/ton), a significant trend break price (about 0.2 Euros/ton), and a normal fluctuation price (about -0.2 Euros/ton). 


\section{Conclusions}

The contributions of the paper may be summarized as follows. The EU ETS carbon future price of maturity December 2013, e.g. DEC13, is decomposed into eight IMFs and one residue. Through the hierarchical clustering method, three components are identified as (i) short term market fluctuations, (ii) effects of significant trend breaks, and (iii) a long-term trend. The trend slowly changing around the long-term mean can basically determine the evolution of the carbon price in the long-term. Significant trend breaks can trigger the sharp downs or ups in carbon prices in the medium-term. Normal market activities can mainly drive the small carbon price fluctuations in the short- term.

The analyses of these three components of the carbon price can provide us some forecasting strategies: we can firstly predict each component based on its own features, such as applying a polynomial function to fit the trend component, a nonlinear forecasting model such as Support Vector Regression or Artificial Neural Network to simulate low frequency component, and a stochastic model such as ARIMA or GARCH to handle high frequency component, and then integrate their results to obtain a final result. How to forecast them based on their concrete implications and data characteristics will be our future research work.

\section{Acknowledgments}

Bangzhu Zhu, Shujiao Ma, Yiming Wei :this work is supported by "the Natural Science Foundation of China (NSFC) (71201010 and 71303174)", "the Ministry of Education of China (11YJC630304)" and "the Natural Science Foundation of Guangdong (S2012010009991).

\section{References}

[1] Zhang YJ, Wei YM. An Overview of Current Research on EU ETS: Evidence from its Operating Mechanism and Economic Effect. Applied Energy 2010; 6: 1804-1814.

[2] Seifert J, Marliese UH, Michael W. Dynamic behavior of CO2 spot prices. Journal of Environmental Economics and Management 2008; 56: 180-194.

[3] Bataller MM, Pardo A., Valor E. CO2 Prices, Energy and Weather. The Energy Journal 2007; 3: 73-92.

[4] Beat H. Allowance price drivers in the first phase of the EUETS. Journal of Environmental Economics and Management 2010; 59: 43-56.

[5] Keppler JH, Bataller MM. Causalities between CO2, electricity, and other energy variables during phase I and phase II of the EU ETS. Energy Policy 2010; 38: 3329-3341.

[6] Montagnoli, A., deVries, F.P., 2010. Carbon trading thickness and market efficiency. Energy Economics, 32:1331-1336.

[7] Feng ZH, Zou LL, Wei YM. Carbon price volatility: Evidence from EU ETS. Applied Energy 2011; 88: $590-598$

[8] Alberola E, Chevallier J, Cheze B. Price Drivers and Structural Breaks in European Carbon Prices 2005-2007. Energy Policy 2008; 2, 787-797.

[9] Chevallier J, Ielpo F, Mercier L. Risk aversion and institutional information disclosure on the European carbon market: a case-study of the 2006 compliance event. Energy Policy 2009; 3: 15-28. 
[10] Chevallier J. Modelling risk premia in $\mathrm{CO}_{2}$ allowances spot and futures prices. Economic Modelling 2010; 27:717-729.

[11]Chevallier J. Wavelet packet transforms analysis applied to carbon prices. Economics Bulletin2011; 31(2):1731-1747.

[12] Creti A, Jouvet PA, Mignon V. Carbon price drivers: Phase I versus Phase II equilibrium? Energy Economics 2012; 34:327-334.

[13] Conrad C., Rittler D., Rotfuß W. Modeling and explaining the dynamics of European Union Allowance prices at high-frequency.Energy Economics 2012; 34: 316-326.

[14] Arouri M.E.H, Jawadi F., Nguyen D.K. Nonlinearities in carbon spot-futures price relationships during Phase II of the EU ETS. Economic Modelling 2012; 29(3):884-892.

[15] Piia A, Markku O, Anne T. Price determination in the EU ETS market: Theory and econometric analysis with market fundamentals. Energy Economics 2013; 36: 380-395.

[16]Crossland J., Li B., Roca E. Is the European Union Emissions Trading Scheme (EU ETS) informationally efficient? Evidence from momentum-based trading strategies. Applied Energy 2013;109: 10-23.

[17] Bredin D, Muckley C. An emerging equilibrium in the EU emissions trading scheme .Energy Economics 2011;33: 353-362.

[18] Lutz BJ, Pigorsch U, Rotfuß W. Nonlinearity in cap-and-trade systems: The EUA price and its fundamentals .Energy Economics 2013;40: 222-232.

[19]Zhu BZ, Wei YM. Carbon price prediction with a hybrid ARIMA and least squares support vector machines methodology. Omega, 2013,(41):517-524.

[20] Huang NE, Shen Z, Long SR. The empirical mode decomposition and the Hilbert spectrum for nonlinear and nonstationary time series analysis. Process of the Royal Society of London 1998; A454: 903-995.

[21] Huang NE, Shen Z, Long SR. A new view of nonlinear water waves: The Hilbert spectrum. Annual Review of Fluid Mechanics 1999; 31: 417-457.

[22] Huang NE, Wu ML, Qu WD. et al. Applications of Hilbert Huang transform to non-stationary financial time series analysis. Applied Stochastic Models in Business and Industry 2003; 19: 245-268.

[23] Zhang X, Lai KK, Wang SY. A new approach for crude oil price analysis based on Empirical Mode Decomposition. Energy Economics 2008; 30: 905-918.

[24] Yu L, Wang SY, Lai KK, et al. A multiscale neural network learning paradigm for financial crisis forecasting. Neurocomputing 2010; 73: 716-72.

[25] Zhu BZ. A Novel Multiscale Ensemble Carbon Price Prediction Model Integrating Empirical Mode Decomposition, Genetic Algorithm and Artificial Neural Network. Energies, 2012, (5):355-370.

[26] Riling G, Flandrin P, Conclaves P. On Empirical Mode Decomposition and its algorithms. IEEE EURASIP Workshop on Nonlinear Signal and Image Processing, Grado (I), 2003.

[27] Zhong C., Miao D., Fränti P. Minimum spanning tree based split-and-merge: A hierarchical clustering method.Information Sciences 2011; 181:3397-3410.

[28] Peel MC, Amirthanathan GE, Pegram GGS, et al. Issues with the application of empirical mode decomposition, In: Zerger, A., Argent, R.M. (Eds.), Modsim 2005 International Congress on Modeling and Simulation, 2005:1681-1687. 
[29] Moghtaderi A, Flandrin P, Borgnat P. Trend filtering via empirical mode decompositions, Computational Statistics \& Data Analysis 2013; 58:114-126. 